\title{
Color Detection of RGB Images Using Python and OpenCv
}

\author{
P. Raguraman ${ }^{1 *}$, A. Meghana ${ }^{2}$, Y. Navya ${ }^{3}$, Sk. Karishma ${ }^{4}$, S. Iswarya ${ }^{5}$ \\ ${ }^{*}$ Assistant Professor, Computer Science and Engineering, Qis College of Engineering and Technology, Ongole, \\ Andhra Pradesh, India \\ ${ }^{2-5}$ Computer Science and Engineering, Qis College of Engineering and Technology, Ongole, Andhra \\ Pradesh, India
}

\begin{abstract}
Article Info

Volume 7, Issue 1

Page Number: 109-112

Publication Issue :

January-February-2021

Article History

The main objective of this application is the methodology for identifying the shades of colors with an exact prediction with their names. A study says, a normal human can able to clearly identify nearly 1 million shades of colors. But in the case of human having "enchroma", could be able to see only $1 \%$ (i.e.10,000 colors) from the normal humans. While painting pictures, a painter needs to identify the color patterns exactly or else the reality of image is not clear.
\end{abstract}

Accepted : 10 Jan 2021

Published : 20 Jan 2021
Keywords : Enchroma, RGB value, OpenCv, pandas

\section{INTRODUCTION}

Before going into the speculations of the project it is important to know the definition of color detection. It is simply the process of identifying the name of any color. It is obvious that humans perform this action naturally and do not put any effort in doing so. While it is not the case for computers.

Human eyes and brain work in co-ordination in order to translate light into color. Light receptors that are present in eyes transmit the signal to the brain which in turn recognizes the color. There is no exaggeration in saying that humans have mapped certain lights with their color names since childhood. The same strategy isuseful in detecting color names in this project.

Three different colors Red, Green and Blue are being tracked by utilizing the fundamentals of computer vision. After successful compilation when we execute the code a window redirectsto the image displayed on it whose path is given as an argument.

Additionally, we obtain the color name of the pixel along with the composition of three different colors red, blue and green values. It is helpful in recognizing colors and in robotics. One of the applications of color detection bycomputer vision is in driver less cars. This system is useful in detecting traffic and vehicle backlights and takes decision to stop, start and continue driving. This also have much application in industry to pick and place different colored object by the robotic arm. Color detection is also used as a tool in various image editing and drawing apps.

\section{METHODS AND MATERIAL}

Image Capture:

The first step is to fetch a high-quality image with resolution. To load an image from a file we use 
Cv2.imread(). Image should be in working directory or full path of the image should be given.

Img=cv2.imread(img path)

\section{Extraction of RGB Colors:}

In this phase, the 3 layered colors are extracted from the input image. All the color images on screens such as televisions, computer, monitors, laptops and mobile screens are produced by the combination of Red, Green and Blue light.

Each primary color takes an intensive value 0 (lowest) to 255 (highest). When mixing 3 primary colors at different intensity levels a variety of colors are produced. For Example: If the intensity value of the primary colors is 0 , this linear combination corresponds to black. If the intensity value of the primary colors is 1 , this linear combination corresponds to white.

Index=[ "color", "color_name", "hex", "R", "G", "B"]

Calculate minimum distance from coordinates:

The minimum distance is calculated by considering moving towards the origin point from all colors to get the most matching color.

The pandas library serves as an important utility to perform various operations on comma-seperated values like pd.read_csv() reads the csv file and loads it into the pandas data frame.

$\mathrm{D}=\operatorname{abs}(\mathrm{R}-\operatorname{int}(\operatorname{csv} \cdot \operatorname{loc}[\mathrm{i}, " \mathrm{R} "]))+$ abs (G-int $(\operatorname{csv} \cdot l o c[i, " G "]))+$ abs $(B-\operatorname{int}(\operatorname{csv} \cdot l o c[i, " B "]))$

Image Display with Shades of Color:

The rectangle window is used to display the image with shades of color. After the double-click is triggered, the RGB values and color name is updated.
To display an image Cv2.imshow () method is used. By using cv2.rectangle and cv2.putText () functions, the color name and its intensity level can be obtained.

text=getColorName $(\mathrm{r}, \mathrm{g}, \mathrm{b})+$ ' $\mathrm{R}={ }^{\prime}+\operatorname{str}(\mathrm{r})+^{\mathrm{G}}={ }^{\prime}+\operatorname{str}(\mathrm{g})+$ ' $\mathrm{B}=$ ' $+\operatorname{str}(\mathrm{b})$.

\section{SYSTEM ARCHITECTURE}

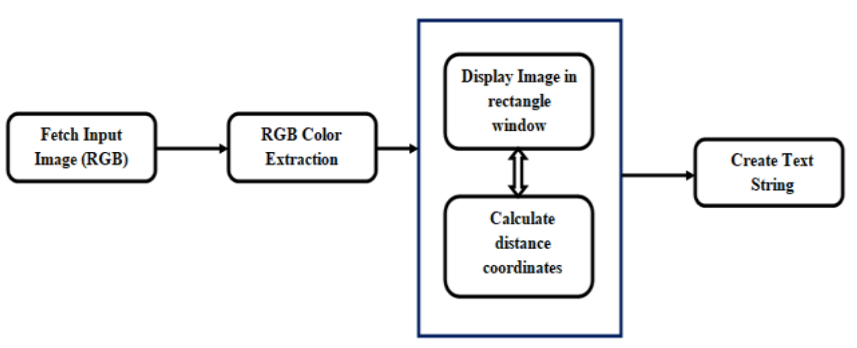

Figure 1 : Architecture Diagram

The above architecture shows the capability for the project. It consists of a well defined sequence diagram that is abstracted from the source code. It leverages the rich capabilities of the technology such as OpenCv library in python.

The above architecture makes the process more efficient based on principles and properties related to each other.As we know that Red, Green and Blue are the primary colors that can be mixed to produce different colors. The present color detection project takes the path of an image as an input and looks for the composition of three different colors red, green and blue in the given image.

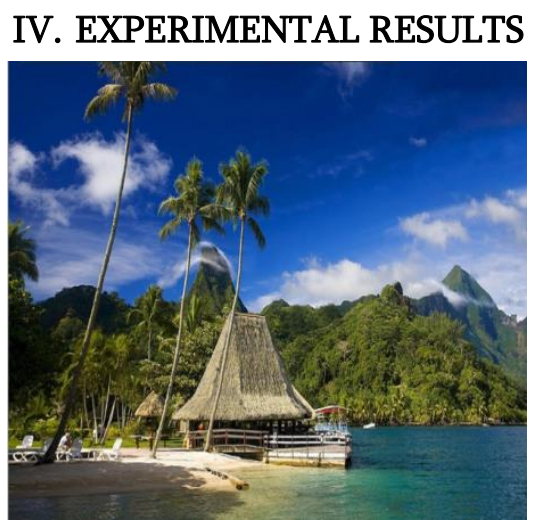

(a) 


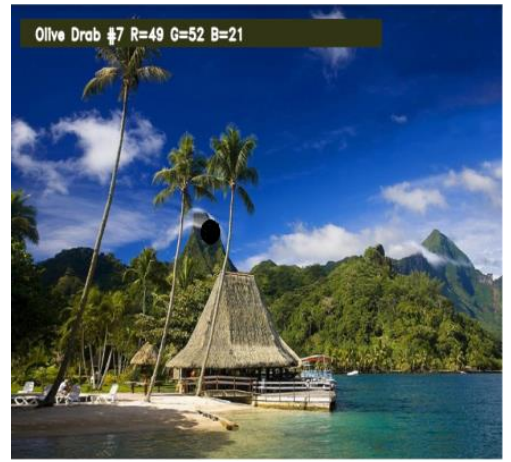

(b)

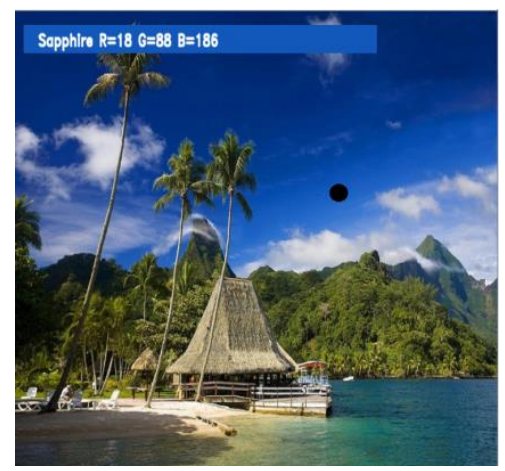

(c)

Figure 2: (a) Original input image of nature (b) Output image with Color intensity RGB values as $\mathrm{R}=49 \mathrm{G}=52 \mathrm{~B}=21$ for Olive Drop (c) Output image with Color intensity $R G B$ values as $R=18 \mathrm{G}=88 \mathrm{~B}=186$ for Sapphire

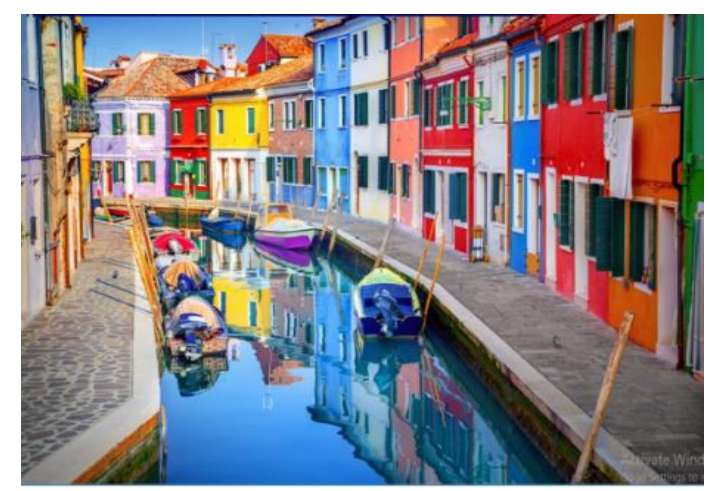

(a)

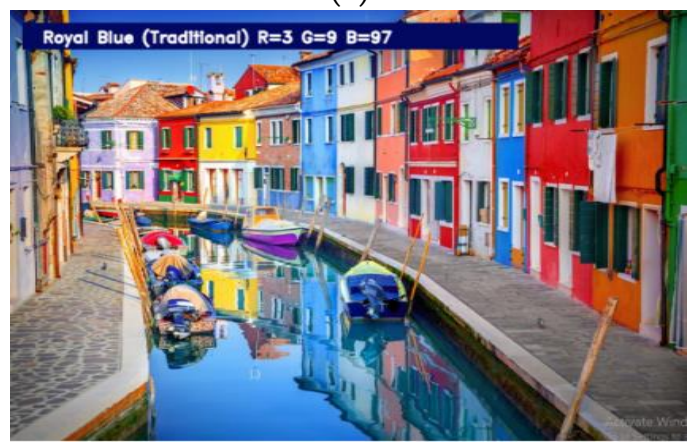

(b)

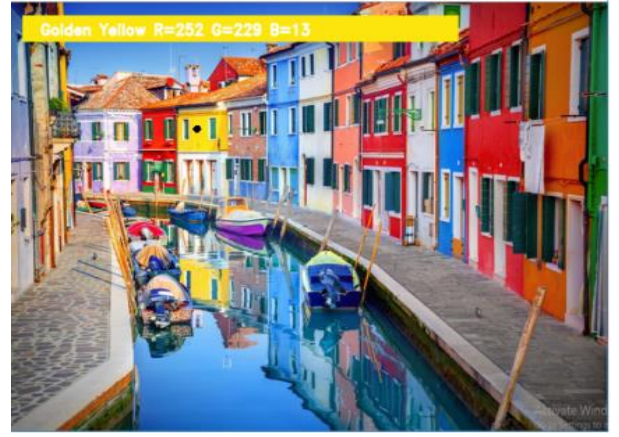

(c)

Figure 3: (a) Original input image of Salt Lake (b) Output image with Color intensity RGB values as $R=3 \mathrm{G}=9 \mathrm{~B}=97$ for Royal Blue (c) Output image with Color intensity RGB values as $R=252 \mathrm{G}=229 \mathrm{~B}=13$ for Golden Yellow

\section{CONCLUSION}

In this paper we defined to get the required color field from an RGB image. In this various steps are implemented using openCv platform. The main positive point of this method is its color differentiation of a mono color.

In the future scope, the detection of the edge detection techniques has different other applications like facial detection, color conversion for grey scale image etc. that can also be implemented.

\section{FUTURE WORK}

In existing system there is no exact color representation of colors with accuracy. In proposed system, we are introducing the CV datasets and according to it the number of shades that can be identified using 865 color names along with their RGB and hex values. Whenever the cursor clicks the image, it automatically shows the RGB shades color values. Proposed system uses OpenCv for sorting of primary colors.

\section{REFERENCES}

[1]. Weiming Hu, Xue Zhou, "Active Counter-Based Visual Tracking by Integrating Colors, Shapes and Motions", IEEE TRANSACTIONS ON 
IMAGE PROCESSING, VOL. 22, NO. 5, MAY 2013

[2]. G.M. Snoek, "Evaluating Color Descriptors for Object and Scene Recognition", IEEE TRANSACTIONS ON PATTERN ANALYSIS AND MACHINE INTELLIGENCE, VOL. 32, NO. 9, SEPTEMBER 2010

[3]. Claudia Nieuwenhuis, "Spatially Varying Color Distributions for Interactive Multi Label Segmentation", IEEE TRANSACTIONS ON PATTERN ANALYSIS AND MACHINE INTELLIGENCE, VOL 35, NO. 5, MAY 2013

[4]. Kok -Meng Lee, "Effects of Classification Methods on Color-Based Feature Detection with Food Processing Applications", IEEE TRANSACTIONS ON AUTOMATION SCIENCE AND ENGINEERING, VOL. 4, NO. 1, JANUARY 2007

[5]. J. Van de Weijer, "Curvature estimation in oriented patterns using curvilinear models applied to gradient vector fields", IEEE TRANS PATTERN ANALYSIS AND MACHINE INTELLIGENCE, VOL. 23, N0. 9, PP. 10351042, APRIL 2001

[6]. Alexander Toet, "Multisource Information Fusion Architectures Algorithms and Applications" SPIE 6947 BELLINGHAM WA USA THE INTERNATIONAL SOCIETY FOR OPTICAL ENGINEERING, 1 12, 2008

\section{Cite this article as :}

P. Raguraman, A. Meghana, Y. Navya, Sk. Karishma, S. Iswarya, "Color Detection of RGB Images Using Python and OpenCv", International Journal of Scientific Research in Computer Science, Engineering and Information Technology (IJSRCSEIT), ISSN : 2456-3307, Volume 7 Issue 1, pp. 109-112, JanuaryFebruary 2021. Available at doi : https://doi.org/10.32628/CSEIT217119 Journal URL : http://ijsrcseit.com/CSEIT217119 